\title{
Rapid Cooling of the Neutron Star in Cassiopeia A Triggered by Neutron Superfluidity in Dense Matter
}

\author{
Dany Page, ${ }^{1}$ Madappa Prakash, ${ }^{2}$ James M. Lattimer, ${ }^{3}$ and Andrew W. Steiner ${ }^{4}$ \\ ${ }^{1}$ Instituto de Astronomía, Universidad Nacional Autónoma de México, Mexico D.F. 04510, Mexico \\ ${ }^{2}$ Department of Physics and Astronomy, Ohio University, Athens, Ohio 45701-2979, USA \\ ${ }^{3}$ Department of Physics and Astronomy, State University of New York at Stony Brook, Stony Brook, New York 11794-3800, USA \\ ${ }^{4}$ Joint Institute for Nuclear Astrophysics, National Superconducting Cyclotron Laboratory and, Department of Physics \\ and Astronomy, Michigan State University, East Lansing, Michigan 48824, USA
}

(Received 29 November 2010; published 22 February 2011)

\begin{abstract}
We propose that the observed cooling of the neutron star in Cassiopeia $\mathrm{A}$ is due to enhanced neutrino emission from the recent onset of the breaking and formation of neutron Cooper pairs in the ${ }^{3} P_{2}$ channel. We find that the critical temperature for this superfluid transition is $\simeq 0.5 \times 10^{9} \mathrm{~K}$. The observed rapidity of the cooling implies that protons were already in a superconducting state with a larger critical temperature. This is the first direct evidence that superfluidity and superconductivity occur at supranuclear densities within neutron stars. Our prediction that this cooling will continue for several decades at the present rate can be tested by continuous monitoring of this neutron star.
\end{abstract}

DOI: 10.1103/PhysRevLett.106.081101

PACS numbers: 97.60.Jd, 95.30.Cq, 26.60.-c

The neutron star in Cassiopeia A (Cas A), discovered in 1999 in the Chandra first light observation [1] targeting the supernova remnant, is the youngest known in the Milky Way. An association with the historical supernova SN 1680 [2] gives Cas A an age of $330 \mathrm{yr}$, in agreement with its kinematic age [3]. The distance to the remnant is estimated to be $3.4_{-0.1}^{+0.3} \mathrm{kpc}$ [4]. The thermal soft x-ray spectrum of Cas A is well fit by a nonmagnetized carbon atmosphere model, with a surface temperature of $2 \times 10^{6} \mathrm{~K}$ and an emitting radius of $8-17 \mathrm{~km}$ [5]. These results raise Cas A to the rank of the very few isolated neutron stars with a well determined age and a reliable surface temperature, thus allowing for detailed modeling of its thermal evolution and the determination of its interior properties [6].

Analyzing archival data from 2000-2009, Heinke and Ho [7] recently reported that Cas A's surface temperature has rapidly decreased from $2.12 \times 10^{6}$ to $2.04 \times 10^{6} \mathrm{~K}$ $[8,9]$. This rate of cooling is significantly larger than expected from the modified Urca ("MU") process $[10,11]$ or a medium modified Urca [12]. It is also unlikely to be due to any of the fast neutrino $(\nu)$ emission processes (such as direct Urca processes from nucleons or hyperons, or $\nu$ emission from Bose condensates or gapless quark matter) since the visible effects of those become apparent over the thermal relaxation time scale of the crust [13]; i.e., 30-100 yr, much earlier than the age of Cas A, and exhibit a slow evolution at later times. We interpret Cas A's cooling within the "minimal cooling" paradigm [14] and suggest it is due to the recent triggering of enhanced neutrino emission resulting from the neutron ${ }^{3} P_{2}$ pairing in the star's core. Our numerical calculations and analytical analysis imply a critical temperature $T_{C} \simeq 0.5 \times 10^{9} \mathrm{~K}$ for the triplet neutron superfluidity.
The essence of the minimal cooling paradigm is the a priori exclusion of all fast $\nu$-emission mechanisms, thus restricting $\nu$ emission to the "standard" MU process and nucleon bremsstrahlung processes [11]. However, effects of pairing, i.e., neutron superfluidity and/or proton superconductivity, are included. At temperatures just below the critical temperature $T_{c}$ of a pairing phase transition, the continuous breaking and formation of Cooper pairs [15], referred to as the "PBF" process, results in an enhanced neutrino emission. Calculations of $T_{c}$ for neutrons, $T_{\mathrm{cn}}$, in the ${ }^{3} P_{2}$ channel relevant for neutron star cores, are uncertain due to unsettled interactions [16] and medium effects which can either strongly suppress or increase the pairing [17]. Consequently, predictions range from vanishingly small to almost $10^{11} \mathrm{~K}$ [14]. The pairing gap is density $(\rho)$ dependent, and the resulting $T_{\mathrm{cn}}(\rho)$ commonly exhibits a bell-shaped density profile. Assuming the neutron star has an isothermal core at temperature $T$, the phase transition will start when $T$ reaches, at some location in the star, the maximum value of $T_{\mathrm{cn}}(\rho)$ : $T_{C} \equiv \max T_{\mathrm{cn}}(\rho)$. At that stage, neutrons in a thick shell go through the phase transition and as $T$ decreases, this shell splits into two shells which slowly drift toward the lower and higher density regions away from the maximum of the bell-shaped profile. If the neutron ${ }^{3} P_{2}$ gap has the appropriate size, $\nu$ emission from the PBF process is an order of magnitude more efficient than the MU process (see Fig. 20 of [14] or Fig. 2 of [18]).

Implications of the size of the neutron ${ }^{3} P_{2}$ pairing gap were considered in [19] with the result that, for $T_{C}<10^{9} \mathrm{~K}$, a neutron star would go through the pairing phase transition at ages ranging from hundreds to tens of thousands of years, accompanied by a short phase of rapid cooling. This phenomenon, illustrated in Fig. 6 of 
[19], closely resembles earlier results, e.g., Fig. 1 of [18] and Fig. 8 of [10]. In Fig. 1, we show similar results of models with the likely surface chemical composition of Cas A from observations [5,7]. The dotted curves show that rapid cooling is strongly dependent on $T_{C}$, a value $\simeq 0.5 \times 10^{9} \mathrm{~K}$ being favored by the present age of Cas A.

We now offer physical insight into the evolution of Cas A based on an analytical model developed in [11]. Assuming the star's core to be isothermal at a temperature $T$, a state reached within a few years after birth, and that the dominant cooling occurs through $\nu$ emission with a luminosity $L_{\nu}$, which is correct for up to several tens of thousands of years, energy balance gives

$$
C_{V} \frac{d T}{d t}=-L_{\nu},
$$

where $C_{V}$ is the star's total specific heat. Neglecting the effects of pairing [20], one can write $C_{V}=C_{9} T_{9}$ with $C_{9} \sim 10^{39} \mathrm{erg} \mathrm{K}^{-1}$ and $T_{9} \equiv T /\left(10^{9} \mathrm{~K}\right)$. Similarly, $L_{\nu}$ from the MU process can be written $L_{\mathrm{MU}}=L_{9} T_{9}^{8}$ with $L_{9} \sim 10^{40} \mathrm{erg} \mathrm{s}^{-1}$. Once triggered, neutrino emission from the PBF process is more efficient than the MU process; i.e., $L_{\mathrm{PBF}}=f L_{\mathrm{MU}}$ with $f \sim 10$ [21]. Values of $C_{9}$ and $L_{9}$ can be found in [11] or from Figs. 11 and 20 of [14]. Considering ages large enough such that $T$ is much smaller than the initial temperature, Eq. (1) gives

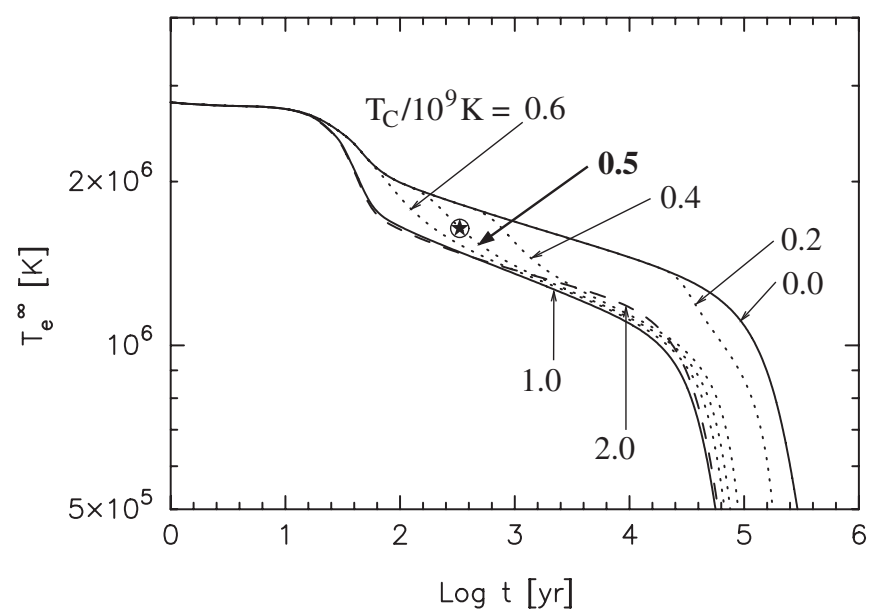

FIG. 1. Redshifted effective temperature $T_{e}^{\infty}$ vs time, of an isolated neutron star for various values of the maximum neutron ${ }^{3} P_{2}$ pairing critical temperature, $T_{C}$ (in units of $10^{9} \mathrm{~K}$ ). The circled star shows the value for Cas A. Results shown are from numerical solutions of the general relativistic energy balance and heat transport equations for a $1.4 M_{\odot}$ star built from the APR equation of state [25]. Refs. [14,19] describe the microphysical inputs used. These results are similar to those in Fig. 6 of [19], but with the difference that the upper layers of the envelope contain light elements of total mass $\Delta M_{\text {light }}=5 \times 10^{-11} M_{\odot}$, compatible with the observation of carbon at the star's surface $[5,7]$. The rapid cooling at ages $\sim 30-100 \mathrm{yr}$ is due to the thermal relaxation of the crust. Thereafter, the stellar interior is isothermal.

$$
T=10^{9} \mathrm{~K}\left(\tau_{\mathrm{MU}} / t\right)^{1 / 6}
$$

with a MU cooling time scale $\tau_{\mathrm{MU}} \equiv 10^{9} C_{9} / 6 L_{9} \sim 1 \mathrm{yr}$. The temperature $T$ will reach $T_{C}$ at a time $t_{C}=\tau_{\mathrm{MU}} / T_{C 9}^{6}$. If this happened recently in the core of Cas A, i.e., at $t_{C} \simeq(0.5-0.9) \times 330 \mathrm{yr}$, one deduces that

$$
T_{C}=\max T_{\mathrm{cn}}(\rho)=10^{9} \mathrm{~K}\left(\tau_{\mathrm{MU}} / t_{C}\right)^{1 / 6} \sim 0.5 \times 10^{9} \mathrm{~K} .
$$

After that moment, $L_{\nu}$ rapidly increases, by a factor $f$, and the solution of Eq. (1) switches to

$$
T=\frac{T_{C}}{\left[1+f\left(t-t_{C}\right) / t_{C}\right]^{1 / 6}}=\frac{T_{C}}{\left[1+\left(t-t_{C}\right) / \tau_{\mathrm{TR}}\right]^{1 / 6}}
$$

with a transit time scale $\tau_{\mathrm{TR}}$ given in terms of the PBF time scale $\tau_{\mathrm{PBF}}=\tau_{\mathrm{MU}} / f$ as

$$
\tau_{\mathrm{TR}} \equiv \frac{t_{C}}{f}=\frac{\tau_{\mathrm{PBF}}}{T_{C 9}^{6}}=\frac{\tau_{\mathrm{MU}}}{f T_{C 9}^{6}} .
$$

With an $f$ of the order of 10 , one can naturally expect a transit time scale of a few decades in the case of Cas A. When $t \gg t_{C}$, the solution in Eq. (4) has the same form as that in Eq. (2), but with the shorter time scale $\tau_{\mathrm{PBF}}$ :

$$
T=10^{9} \mathrm{~K}\left(\tau_{\mathrm{PBF}} / t\right)^{1 / 6}=10^{9} \mathrm{~K}\left(\tau_{\mathrm{MU}} / f t\right)^{1 / 6} .
$$

The above evolution is depicted schematically in the left panel of Fig. 2.

For comparison with observations, we convert the internal $T$ to the observed effective $T_{e}$ using (see, e.g., [11])
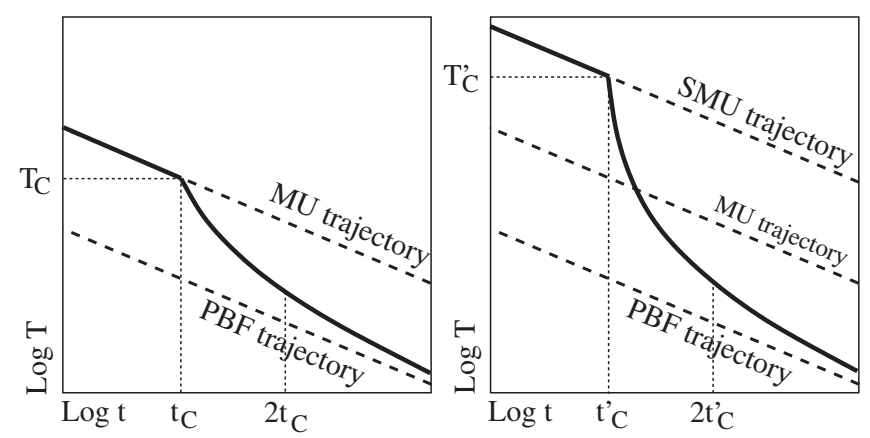

FIG. 2. Schematic evolution of the internal temperature $T$ of a neutron star (solid curves). Left panel: Initially, $T$ follows the upper modified Urca (MU) trajectory, Eq. (2), until $T$ reaches $T_{C}$ at time $t_{C}$ when the pair breaking and formation (PBF) process turns on. Thereafter, $T$ rapidly transits, on an initial time scale $\tau_{\mathrm{TR}}=t_{C} / f$, Eq. (4), toward the lower PBF trajectory, Eq. (6). Empirically, the rapid transition lasts up to a time $\lesssim 2 t_{C}$. Right panel: Evolution using a superconductivity suppressed modified Urca (SMU) rate. For the transit to start at a time $t_{C}^{\prime} \approx t_{C}$ the trajectory requires a $T_{C}^{\prime}$ larger than $T_{C}$ of the left panel. The early transit has a shorter time scale $\tau_{\mathrm{TR}}^{\prime}=t_{C}^{\prime} / f^{\prime}$ with $f^{\prime}>f$, but the late time evolution is almost identical to that of the left panel. The left (right) panel corresponds to models of Fig. 1 (Figs. 3 and 4). 


$$
T_{e} \simeq T_{e 0}\left(T / 10^{8} \mathrm{~K}\right)^{\beta} \mathrm{K},
$$

where $T_{e 0} \sim 10^{6} \mathrm{~K}$ and $\beta \sim 0.5$. The evolution of $T_{e}$ is hence similar to that of $T$, and the internal cooling curves of Fig. 2 map onto analogous models of Figs. 1, 3, and 4. The scale $T_{e 0}$ and the exponent $\beta$ in Eq. (7) both depend on the chemical composition of the envelope. The presence of light elements, e.g., $\mathrm{H}, \mathrm{He}, \mathrm{C}$, and/or $\mathrm{O}$, increases $T_{e 0}$ and reduces $\beta$ compared to the case of heavy elements, e.g., $\mathrm{Fe}$, depending on the total mass $\Delta M_{\text {light }}$ of light elements [22].

Using Eq. (7), the slope $s=d \log _{10} T_{e} / d \log _{10} t$ of the transit cooling curve from Eq. (4) is

$$
s=\beta \frac{d \log _{10} T}{d \log _{10} t}=-\frac{\beta}{6} \frac{f t / t_{C}}{1+f\left(t-t_{C}\right) / t_{C}},
$$

whereas the slopes of the asymptotic trajectories, Eqs. (2) and (6), are both $s=-\beta / 6 \sim-1 / 12$. As long as $t$ is only slightly larger than $t_{C}$, the transit slope is larger than those of the asymptotic trajectories by a factor $\sim f$. The observed slope over a $10 \mathrm{yr}$ interval is $s_{\text {obs }} \simeq-1.4$. Note, however, that the model " 0.5 " of Fig. 1 does not exhibit such a large slope. We are thus led to investigate the origin of the rapidity of Cas A's cooling.

Several factors influence the rapidity of the transit phase. First, $L_{\mathrm{PBF}}$ depends on the shape of the $T_{\mathrm{cn}}(\rho)$ curve. A weak $\rho$ dependence, i.e., a wide $T_{\text {cn }}(\rho)$ curve, results in a thicker PBF neutrino emitting shell and a larger $L_{\mathrm{PBF}}$ than a strong $\rho$ dependence. Second, the $T$ dependence of $T_{e}$, i.e., the parameter $\beta$ in Eq. (7), also affects the slope in Eq. (8). Third, protons in the core will likely exhibit superconductivity in the ${ }^{1} S_{0}$ channel. Most calculations of the proton critical temperature, $T_{\mathrm{cp}}(\rho)$, are larger than $T_{\mathrm{cn}}(\rho)$ at low

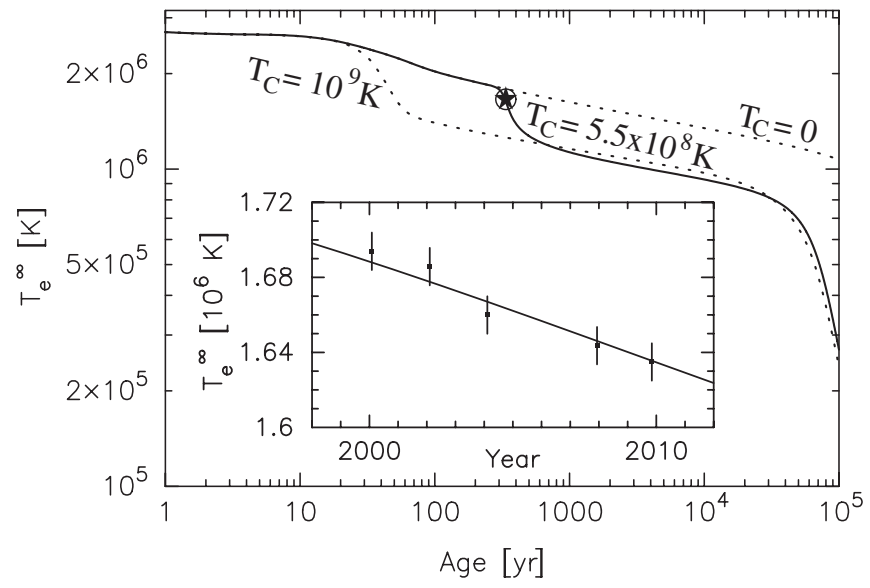

FIG. 3. A typical good fit to Cas A's rapid cooling for a $1.4 M_{\odot}$ star, built from the EOS of APR [25] with an envelope mass $\Delta M_{\text {light }}=5 \times 10^{-13} M_{\odot}$. The two dotted curves, with indicated values of $T_{C}$, are to guide the eye. The three models have a proton " $S_{0}$ gap from [26] (the model "CCDK" in [14]) which results in the entire core being superconducting. The insert shows a comparison of our results with the five data points of [7] along with their $1 \sigma$ errors. densities. Proton superconductivity suppresses the MU process in a large volume of the core at a very early age, reducing $L_{\mathrm{MU}}$ [23]. In our analytical model, this reduction translates to a lower $L_{9}$ and, hence, to a larger $f$. The analytical model as well as our calculations reveal that proton superconductivity significantly accelerates cooling during transit and results in a large slope. This feature, essential to account for Cas A's cooling rate, is illustrated in the right panel of Fig. 2.

By varying the relevant physical ingredients, such as the density range of proton ${ }^{1} S_{0}$ superconductivity, the shape of the $T_{\mathrm{cn}}(\rho)$ curve, the chemical composition of the envelope, and the star's mass, many models can reproduce the average observed $T_{e}$ of Cas A. These models yield slopes ranging from $\sim-0.1$ (no rapid cooling and no constraint on $T_{C}$ ) up to -2 . A typical good fit to the rapid cooling of Cas A is shown in Fig. 3, where the large slope results from the strong suppression of $L_{\mathrm{MU}}$ by extensive proton superconductivity. Figure 4 demonstrates that the result $T_{C} \simeq 0.5 \times 10^{9} \mathrm{~K}$ does not depend on the star's mass, but that the slope during the transit is very sensitive to the extent of proton superconductivity. Models successful in reproducing the observed slope require superconducting protons in the entire core. Although spectral fits [5] seem to indicate that Cas A has a larger than canonical mass $\left(1.4 M_{\odot}\right)$, a recent analysis [6] indicates compatibility, to within $3 \sigma$, with a smaller mass, $1.25 M_{\odot}$. The need for extensive proton superconductivity to reproduce the large observed slope favors moderate masses unless superconductivity extends to much higher densities than current models predict (see, e.g., Fig 9 in [14] for a large sample of current models).

The inferred $T_{C} \simeq 0.5 \times 10^{9} \mathrm{~K}$, either from Figs. 1, 3, and 4 or from Eq. (3), appears quite robust and stems from the small exponent in the relation $T_{C} \propto\left(C_{9} L_{9}^{-1} t_{C}^{-1}\right)^{1 / 6}$. Assuming $L_{9}$ is not very strongly affected by proton

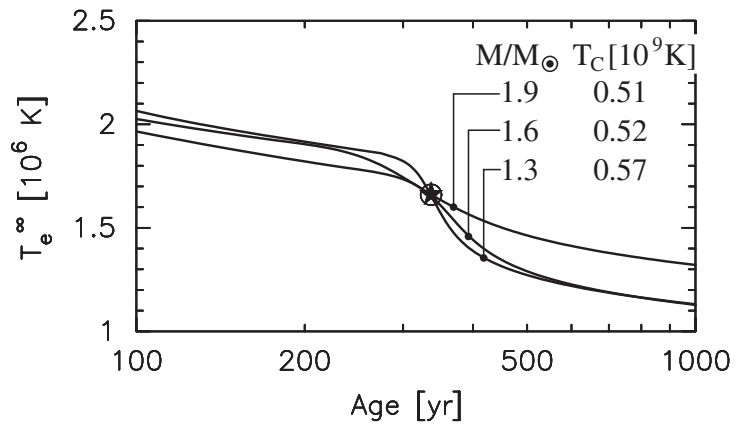

FIG. 4. Cooling curves with different masses and values of $T_{C}$ as indicated. For the $1.9 M_{\odot}$ star, $\Delta M_{\text {light }}=5 \times 10^{-11} M_{\odot}$. For the other two masses shown, $\Delta M_{\text {light }}=5 \times 10^{-13} M_{\odot}$. The assumed proton ${ }^{1} S_{0}$ gap is the same as in Fig. 3. The slopes, at the current age of Cas A, are $-1.4,-0.9$, and -0.5 for the 1.3, 1.6 , and $1.9 M_{\odot}$ models, respectively: the decrease, with increasing mass, directly reflects the decrease of the core's fractional volumes in which protons are superconducting. 
superconductivity, $T_{C}$ is mostly determined by $t_{C}$ which, given the briefness of the rapid cooling transit phase, cannot be much smaller than the age of Cas A. On the other hand, the rapidity of the cooling, i.e., the slope $s$, is predominantly controlled by proton superconductivity reflected in the suppression of $L_{\mathrm{MU}}$. We note, however, that if the proton ${ }^{1} S_{0}$ gap extends to much higher densities than current theoretical models indicate, suppression of $L_{\mathrm{MU}}$ could be larger than considered here. This suppression can be up to a factor $\sim 50$; thus, $T_{C}$ could almost reach $10^{9} \mathrm{~K}[24]$.

Although we have assumed the minimal cooling paradigm [14], our results remain valid in case some fast $\nu$ process is allowed. Any of these processes is so efficient that the high observed $T_{e}^{\infty}$ of Cas A implies that it has been strongly suppressed at a very young age (likely by pairing of one of the participating particles) and rendered inoperative [11]. In such a case, $L_{\nu}$, prior to the onset of the neutron ${ }^{3} P_{2}$ phase transition, was dominated by the MU and nucleon bremsstrahlung processes, exactly as in the minimal cooling paradigm, and the results of our present analysis are not altered.

Our value of $T_{C}$ deduced from Cas A's cooling is compatible with the requirement $T_{C} \gtrsim 0.5 \times 10^{9} \mathrm{~K}$ inferred from the minimal cooling paradigm described in [19]. We predict that Cas A will continue to cool for several decades with an almost constant rate, in contrast to two other interpretations [7]; a relaxation from a recent sudden energy release deep in the crust or a low level accretion from a fossil disc that imply that the observed cooling is episodic and that the light curve should flatten out soon. Distinguishing among these possibilities and our model will be possible by continuous monitoring of the thermal evolution of Cas A.

D. P.'s work is supported by a grant from UNAMDGAPA No. IN122609. M. P. and J. M. L. acknowledge research support from the U.S. DOE grants DE-FG0293ER-40756 and DE-AC02-87ER40317, respectively. A.W.S. is supported by the Joint Institute for Nuclear Astrophysics under NSF-PFC grant PHYS 08-22648, by NASA ATFP grant NNX08AG76G, and by the NSF under grant number PHY-08-00026.

[1] H. Tananbaum, IAU Circ. 7246, 1 (1999); J. P. Hughes et al., Astrophys. J. Lett. 528, L109 (2000).

[2] W. B. Ashworth, Jr., Journal for the History of Astronomy 11, 1 (1980)
[3] R. A. Fesen et al., Astrophys. J. 645, 283 (2006).

[4] J.E. Reed et al., Astrophys. J. 440, 706 (1995).

[5] W. C. G. Ho and C. O. Heinke, Nature (London) 462, 71 (2009).

[6] D. G. Yakovlev et al., arXiv:1010.1154 [Mon. Not. R. Astron. Soc. (to be published)].

[7] C. O. Heinke and W. C. G. Ho, Astrophys. J. Lett. 719, L167 (2010).

[8] Recently, a new observation of Cas A was reported which confirmed this evolution [9].

[9] P.S. Shternin et al., arXiv:1012.0045 [Mon. Not. R. Astron. Soc. Lett. (to be published)].

[10] D. G. Yakovlev and C. J. Pethick, Annu. Rev. Astron. Astrophys. 42, 169 (2004).

[11] D. Page, U. Geppert, and F. Weber, Nucl. Phys. A777, 497 (2006).

[12] H. Grigorian and D. N. Voskresensky, Astron. Astrophys. 444, 913 (2005).

[13] J. M. Lattimer et al., Astrophys. J. 425, 802 (1994).

[14] D. Page et al., Astrophys. J. Suppl. Ser. 155, 623 (2004).

[15] E. Flowers, M. Ruderman, and P. Sutherland, Astrophys. J. 205, 541 (1976); D. N. Voskresensky and A. V. Senatorov, Sov. J. Nucl. Phys. 45, 411 (1987).

[16] M. Baldo et al., Phys. Rev. C 58, 1921 (1998).

[17] A. Schwenk and B. Friman, Phys. Rev. Lett. 92, 082501 (2004); V. A. Khodel et al., Phys. Rev. Lett. 93, 151101 (2004).

[18] M.E. Gusakov et al., Astron. Astrophys. 423, 1063 (2004).

[19] D. Page et al., Astrophys. J. 707, 1131 (2009).

[20] Proton pairing at $T>T_{C}$ is likely but has a small, at most $20 \%$, effect on $C_{V}$. Neutron superfluidity, at $T<T_{C}$, has a larger effect. Comparison of our analytical model with the numerical results of Figs. 1, 3, and 4 shows that the approximation $C_{V} \propto T$ is reasonable.

[21] The neutrino emissivity from the PBF process, once integrated over the core volume, is reasonably well approximated by a $T^{8}$ law $[14,18]$.

[22] A. Y. Potekhin, G. Chabrier, and D. G. Yakovlev, Astron. Astrophys. 323, 415 (1997).

[23] The proton ${ }^{1} S_{0}$ pairing phase transition also induces a $\mathrm{PBF}$ process. It is, however, suppressed by a factor $\left(p_{F} / m\right)^{2}$ compared to the triplet case and, hence, not sufficient to explain the observed cooling of Cas A.

[24] Such values of $T_{\mathrm{cp}}(\rho)$ in modeling Cas A have been suggested and employed in [9] which reports $T_{C} \simeq$ $0.7-0.9 \times 10^{9} \mathrm{~K}$, but otherwise reaches similar conclusions as we here obtain.

[25] A. Akmal, V.R. Pandharipande, and D. G. Ravenhall, Phys. Rev. C 58, 1804 (1998).

[26] J. M. C. Chen et al., Nucl. Phys. A555, 59 (1993). 